\title{
ASSESMENT OF 28 DAYS REPEATED ADMINSTERATION TOXICITY PROFILE OF DEXAMETHASONE PALMITATE INJECTION
}

\author{
Ganesh N. Sharma*, Shaili Rasania, Paresh Dadhaniya, Chintan Patel, Kapil Vachhani \\ School of Pharmaceutical Sciences, Jaipur National University, Jaipur- 302017 \\ Dept. of Preclinical Study, Cadila Pharmaceutical Pvt. Lt., Gujrat, India
}

\begin{abstract}
Although, the dexamethasone have been used for the managements, against various ailments as single API or in combination with other drugs as acetate or palmitate salt. However, Dexamethasone as palmitate salt has not been investigated so far for sub acute toxicity on intravenous administration. Therefore, an attempt has been made to investigate sub-acute toxicity of dexamethasone palmitate injection. The drug was administered in experimental animals at different dose levels (0.07, 0.18 and $0.44 \mathrm{mg} / \mathrm{kg}$, b.w.), for 28 days, and alteration in biochemical and haematological parameters was recorded along with histological examinations. The result data revealed that intravenous administration of dexamethasone palmitate does not causes any significant alteration in experimental animals examined at different levels. The observed changes were either well established adverse effects of Dexamethasone or were in laboratory reference range limit. The intravenous administered doses of drug were found safer, although somewhat adverse reactions may be observed at higher dose level (s).
\end{abstract}

Key Words: Dexamethasone Palmitate, Intravenous, Toxicity, Biochemical

Corresponding Author

Dr. Ganesh N. Sharma

School of Pharmaceutical Sciences,

Jaipur National University, Jaipur- 302017

Email: ganeshmph@gmail.com

Mob: +91-9950664580 


\section{INTRODUCTION}

Corticosteroids are the substances secreted from cortical region of suprarenal gland, named as they are the substances with steroidal structure and are categorised as glucocorticoids and mineralocorticoids [1]. The basic chemical structure- cyclopentano-perhydro-phenanthrene or steroid ring is incorporated in them [2]. Steroids being small lipophilic molecules enter their target cells by simple diffusion [3].

Glucocorticoids are widely used as therapeutic agents against a number of disorders including, inflammation, autoimmune diseases and cancers [4]. Based on duration of action glucocorticoids are further subcategorised as; short acting (Hydrocortisone, Cortisone), Intermediate acting (Prednisolone, Methylprednisolone) and Long acting (Dexamethasone and Betamethasone) [5].

Dexamethasone has been found effective against the chronic lung disorder in prematurely born infant [6,7], inflammatory [8] and many others disorders. Dexamethasone is being marketed as single API or in combination with other drugs as acetate salt under different brand names. The palmitate salt of dexamethasone is white milky colored, lipophilic steroidal drug.

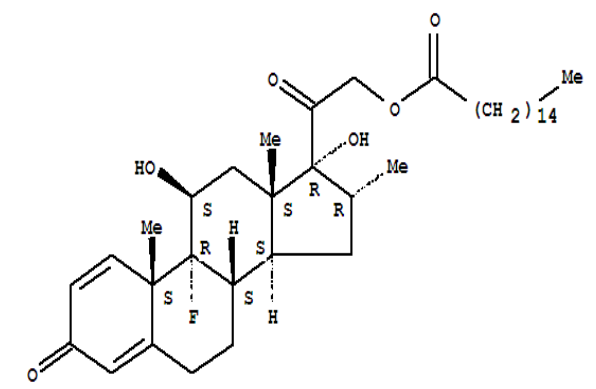

Chemical structure of Dexamethasone Palmitate [9]

The fundamental aim of any toxicological study is to obtain biological information, indicative of toxicity that is reliable, reproducible and dose related and which can be interpreted and/or extended to health risk of humans. Toxicity can be categorised as acute toxicity (caused by single and short term exposure of agent and seen within 24 hours of exposure) [10], sub acute toxicity (caused by repeated mild exposure for several hours or even days and occurs within few hours or days) [11], sub chronic toxicity (caused by repeated exposure to the toxic substance $t$ for a longer period of time which may extend from several days to weeks) [12] and chronic toxicity (usually caused by cumulative toxicity of agents) [13]. The aim of present study was to assess sub acute toxicity, produced by the of dexamethasone palmitate, on intravenous administration in experimental animals.

\section{MATERIAL AND METHODS}

\section{Chemical and Reagents}

Dexamethasone palmitate injection ( $2 \mathrm{ml}$ ampoule) was supplied as gift sample from Cadila Pharmaceuticals Pvt. Ltd (Dholka). All the chemicals and reagents used in study were of analytical grade. Glycerin (Mistral Labs), Ethanol (Merck), Egg lecithin (Maritz Mayer), Oleic acid (Sadtler) and water for injection (Gibco) were incorporated in study.

\section{Assessment of Sub acute toxicity}

Animals: The healthy wistar rats (180-220g) were used in study. They were housed in polypropylene cages and were left for seven days for acclimatization to animal room, maintained under controlled conditions of $12-14$ hours light and dark cycle, $25^{\circ} \mathrm{C} \pm 2{ }^{\circ} \mathrm{C}$ and relative humidity $30-70 \%$. The animals were kept on standard pellet diet and water ad libitum. Prior to study, the protocol was submitted to Institutional Animal Ethics Committee (IAEC) and The Committee for 
the Purpose and Supervision of Experiments on Animals (CPCSEA), and was approved viz. number CPL-12-020.

Dose selection and grouping of animals: The doses of dexamethasone palmitate, to be administered in experimental animals were selected on the basis of the maximum recommended human dose (MRHD) information and were converted to rat dose using body surface area conversion factor. Additional support was taken from published literature on preclinical safety studies of these drugs [14].

The animals were divided into four groups, each consisting of six. Group I received vehicle (mixture of soya bean oil, egg lecithin, sodium oleate, glycerol and water for injection) and served as control. Group II, III and IV received dexamethasone palmitate at the dose of $0.07,0.18$ and 0.44 $\mathrm{mg} / \mathrm{kg}$, b.w. respectively.

The dexamethasone palmitate was injected intravenously through the tail vein using an intubation needle (26 gauge), fitted into a polypropylene disposable syringe, daily, once a day upto 28 days. The study was carried out on both gender animals separately; i.e. male and female, to minimise the physiological varations on study result.

\section{Evaluation of Effects of Dexamethasone Palmitate on animals}

Evaluation of clinical signs: All the experimental animals were observed and evaluated for various clinical signs (including general physiological characteristics, functional changes and behavioral changes).

Mortality and morbidity: All the experimental animals were observed daily for any mortality and morbidity throughout the experimental period.

Body weight: Weight of each animal was recorded one day before the initiation of the treatment (Day 0) and at weekly intervals throughout the period of the study. The last body weight was recorded one day prior to blood collection.

Feed consumption: The quantity of the feed offered was based on the requirement of the animals in each cage and the same was recorded. The leftover of the feed was recorded weekly once. The last feed left over was taken on day 28 of the study exactly before keeping the animals for fasting.

The feed intake of each rat was calculated as:

Feed intake of 6 animals/cage/week $=$

Total quantity of feed input $(\mathrm{g})$ - Feed left over on last during that week $(\mathrm{g})$

Feed intake of individual animal/week $=$ Feed intake of 6 animals per week $/ 6$

Feed intake by individual animal/day $=$ Feed intake of individual animal per week $/ 7$

Collection of blood sample: After completion of the treatment period of 28 days, blood was collected from all animals under diethyl ether anaesthesia on the next day. Approximately 0.7-1.0 $\mathrm{ml}$ of blood samples were collected from retro-orbital sinus and were transferred to $1.5 \mathrm{ml}$ capacity eppendroff containing EDTA solution as an anticoagulant. From this a part of blood was utilized for haematological analysis and remaining was centrifuged to proceed for the biochemical analysis.

Determination of Effect on organ weight and necropsy: The animals were euthanized on day 29 using carbon dioxide asphyxiation. Post mortem necropsy findings were made by systemic approach (i.e. gross changes in organ size, shape and any visible lesions). The organs were collected, cleaned using filter paper and then weighed using analytical balance (Mettler Toledo).

Histopathological studies: Individual organs from sacrificed animals were isolated and were preserved in $10 \%$ neutral buffer formalin solution. Tissues were made free from fatty tissues and 
other unwanted attachments, with forceps and scissors under trimming hood. For chemical fixation, the samples were transferred to a cassette. These cassettes were immersed in multiple baths of progressively more concentrated ethanol, to dehydrate the tissue, followed by xylene and finally in liquid paraffin. The processed tissues were taken out of the cassette and set in a mould. Sections were cut at 5-7 $\mu$ thickness with microtome machine on the glass slide. All the slides were stained with haematoxylin and eosin stain in automatic staining machine (LEICA AUTO STAINER XL). The stained slides were observed under microscope and histological lesions were recorded.

Statistical Analysis: The result data are expressed as mean \pm standard error of mean (SEM) and statistical analysis was done using one way analysis of variance (ANOVA) followed by the student's ' $t$ ' test.

\section{RESULT AND DISCUSSION}

Effect on Clinical signs: No apparent clinical signs of toxicity (Cage side changes, functional changes, autonomic changes and behavioural changes) were noticed during the treatment period of 28 days, belonging to group I to IV in both males and females. Thus, it can be said that dexamethasone palmitate does not produce any toxicity up to the dose of $0.44 \mathrm{mg} / \mathrm{Kg}$ of body weight.

Mortality and Morbidity: No morbidity and mortality was observed and all animals of all experiment groups survived throughout the treatment period of 28 days. Thus, it can be said that dexamethasone palmitate does not produce any toxicity up to the dose of $0.44 \mathrm{mg} / \mathrm{Kg}$ of body weight.

Effect on body weight: The result data are given in table1. Although, all the animals were found to increase in weight with respect of time, yet the increase in weight was found to be affected in dexamethasone treated group as compared to the normal. The significant effect $(p<0.05)$ was observed in animals of group III and IV as compared to normal. The impact on increase in body weight starts just after initial dose, and reached towards higher value with sustained exposure of Dexamethasone Palmitate. The reduction in increase in body weight of animals might be due to inhibition of growth hormone and may be because of increase synthesis and release of leptin and CRH [15-18] or can be attributed to the dexamethasone-induced reduction in nitrogen retention and elevated protein catabolism [19].

Table 1: Effect of Dexamethasone Palmitate on weekly body weight (gm) in rats

\begin{tabular}{cllllllll}
\hline \multirow{2}{*}{ aay } & \multicolumn{2}{c}{ Group I } & \multicolumn{2}{c}{ Group II } & \multicolumn{2}{c}{ Group III } & \multicolumn{2}{c}{ Group IV } \\
\cline { 2 - 8 } & Male & Female & Male & Female & Male & Female & Male & Female \\
\hline 0 & $214.0 \pm 7.8$ & $157.7 \pm 4.9$ & $213.2 \pm 7.9$ & $157.5 \pm 3.9$ & $212.7 \pm 7.5$ & $158.3 \pm 3.4$ & $212.3 \pm 7.1$ & $157.7 \pm 3.5$ \\
8 & $234.5 \pm 7.2$ & $169.9 \pm 5.0$ & $218.5 \pm 5.3$ & $165.0 \pm 6.1$ & $215.3 \pm 8.2$ & $169.2 \pm 7.7$ & $216.3 \pm 5.3$ & $165.5 \pm 6.4$ \\
15 & $272.3 \pm 7.6$ & $193.8 \pm 6.3$ & $235.2 \pm 4.5$ & $172.3 \pm 6.3$ & $232.3 \pm 7.7$ & $175.0 \pm 7.0$ & $231.0 \pm 4.6$ & $168.0 \pm 8.4$ \\
21 & $297.8 \pm 7.2$ & $206.2 \pm 6.1$ & $260.4 \pm 5.3$ & $186.8 \pm 6.9$ & $235.6 \pm 9.7$ & $173.2 \pm 4.4$ & $233.7 \pm 6.4$ & $170.2 \pm 6.6$ \\
28 & $299.3 \pm 8.5$ & $212.8 \pm 5.2$ & $273.2 \pm 5.5$ & $198.0 \pm 8.2$ & $239.2 \pm 11.8$ & $179.7 \pm 4.9$ & $237.8 \pm 6.2$ & $173.3 \pm 5.3$ \\
\hline
\end{tabular}

$>$ Values are given as mean + SEM for groups of 6 animals each.

$>*_{\mathrm{p}}<0.05$ as compared to vehicle control was considered as significant.

Effect on feed Consumption: The administration of Dexamethasone Palmitate injection does not found to cause any significant change in diet capacity in experimental animals. All the animal groups were found normal towards their regular diet, which indicate that dexamethasone palmitate does not produce any adverse effect on feed consumption in animals up to the dose of $0.44 \mathrm{mg} / \mathrm{Kg}$ of body weight. The data are given in Table. 2 
Table.2: Effect of Dexamethasone Palmitate on feed consumption in wistar rats

\begin{tabular}{ccccccccc}
\hline & \multicolumn{2}{c}{ Group I } & \multicolumn{2}{c}{ Group II } & \multicolumn{2}{c}{ Group III } & \multicolumn{2}{c}{ Group IV } \\
Day & Male & Female & Male & Female & Male & Female & Male & Female \\
\hline 8 & 19.79 & 15.33 & 19.38 & 16 & 18.50 & 15.67 & & 14.81 \\
15 & 20.36 & 17.05 & 19.19 & 17.29 & 18.93 & 15.83 & 18.21 & 15.21 \\
21 & 19.81 & 17.6 & 18.40 & 15.48 & 16.98 & 14.33 & 18.45 & 13.98 \\
28 & 18.62 & 15.12 & 17.76 & 13.52 & 17.55 & 13.21 & 16.07 & 13.29 \\
\hline
\end{tabular}

$>$ Values are given as mean as gram of feed/rat/day

$>{ }^{*} \mathrm{p}<0.05$ as compared to vehicle control was considered as significant.

\section{Effect of Dexamethasone Palmitate on haematological parameters}

The results of haematological study are given in table 3 and 4 for male and female animals; respectively. The result data shows that dexamethasone palmitate causes an increase in RBCs count, which establishes the previously reported effect of dexamethasone that it stimulates RBCs production by an effect on erythropoietin production $[20,21]$ and retarding erythrophagocytosis [22]. A slight elevated levels of neutrophills and monocyte was also found but, these values were in normal laboratory range. Some researchers have demonstrated that corticosteroids treatment causes the neutrophils to detach from endothelial cells of blood vessels and inhibit the rate of removal from vascular compartment [23] and apoptosis of neutrophils [24]. At the same time, lymphocyte count reduced in dose dependant manner in drug treated animals. All above mentioned cellular count changes were in reference laboratory range and were not significant, hence were not counted as adverse effect. No significant alteration in packed cell volume (PCV), mean corpuscular volume (MCV), mean corpuscular haemoglobin $(\mathrm{MCH})$, mean corpuscular haemoglobin concentration (MCHC), haemoglobin ( $\mathrm{Hb}$ ), platelets, white blood corpuscles (WBCs) including, eosinophils and basophils was recorded in dexamethasone palmitate treated animals.

Table 3: Effect of Dexamethasone Palmitate on haematological parameters in male wistar rats

\begin{tabular}{llcccc}
\hline Parameters & Group I & Group II & Group III & Group IV \\
\hline RBC & X10 ${ }^{6} \mu \mathrm{L}$ & $7.89 \pm 0.12$ & $8.42 \pm 0.07$ & $8.53 \pm 0.06$ & $8.69 \pm 0.16$ \\
PCV & $\%$ & $47.22 \pm 0.79$ & $47.63 \pm 0.44$ & $47.93 \pm 0.26$ & $47.92 \pm 0.83$ \\
MCV & $\mathrm{Fl}$ & $57.58 \pm 0.19$ & $56.60 \pm 0.72$ & $56.20 \pm 0.29$ & $57.63 \pm .044$ \\
MCH & $\mathrm{Pg}$ & $18.17 \pm 0.39$ & $17.67 \pm 0.25$ & $18.17 \pm 0.07$ & $18.33 \pm 0.17$ \\
MCHC & $\mathrm{g} / \mathrm{Dl}$ & $31.00 \pm 0.30$ & $31.18 \pm 0.20$ & $32.30 \pm 0.22$ & $31.82 \pm 0.14$ \\
HGB & $\mathrm{g} / \mathrm{Dl}$ & $15.33 \pm 0.35$ & $14.85 \pm 0.16$ & $15.48 \pm 0.16$ & $15.25 \pm 0.21$ \\
PLT & $\mathrm{X} 10^{3} \mu \mathrm{L}$ & $1296.50 \pm 90.78$ & $1282.83 \pm 69.87$ & $1199.83 \pm 28.91$ & $1266.83 \pm 45.56$ \\
WBC & $\mathrm{X} 10^{3} \mu \mathrm{L}$ & $17.14 \pm 1.30$ & $16.48 \pm 1.79$ & $16.74 \pm 0.80$ & $17.68 \pm 0.66$ \\
Differe & Neutrophils & $30.00 \pm 5.51$ & $32.83 \pm 2.75$ & $33.00 \pm 2.96$ & $37.48 \pm 0.98$ \\
ntial & Lymphocytes & $64.87 \pm 6.01$ & $62.05 \pm 2.40$ & $61.28 \pm 3.15$ & $56.03 \pm 1.18$ \\
WBCs & Monocytes & $1.78 \pm 0.16$ & $2.17 \pm 0.32$ & $2.47 \pm 0.19$ & $2.97 \pm 0.30$ \\
$(\%)$ & Eosinophils & $0.45 \pm 0.04$ & $0.45 \pm 0.08$ & $0.49 \pm 0.09$ & $0.39 \pm 0.06$ \\
& Basophils & $0.42 \pm 0.05$ & $0.37 \pm 0.06$ & $0.35 \pm 0.03$ & $0.37 \pm 0.02$ \\
\hline
\end{tabular}

$>$ Values are given as mean + SEM for groups of 6 animals each.

$>*_{\mathrm{p}}<0.05$ as compared to vehicle control was considered as significant. 
Table 4: Effect of Dexamethasone Palmitate on hematological parameters in female rats

\begin{tabular}{cccccc}
\hline \multicolumn{2}{c}{ Parameters } & Group I & Group II & Group III & Group IV \\
\hline RBC & X10 ${ }^{6} \mu \mathrm{L}$ & $7.16 \pm 0.13$ & $7.44 \pm 0.15$ & $7.57 \pm 0.22$ & $7.68 \pm 0.12$ \\
PCV & $\%$ & $42.33 \pm 0.74$ & $43.78 \pm 0.60$ & $42.55 \pm 1.11$ & $43.70 \pm 0.97$ \\
MCV & $\mathrm{Fl}$ & $57.17 \pm 0.56$ & $58.18 \pm 0.49$ & $57.03 \pm 0.25$ & $57.47 \pm 0.62$ \\
MCH & $\mathrm{Pg}$ & $18.38 \pm 0.29$ & $18.50 \pm 0.30$ & $17.70 \pm 0.25$ & $18.02 \pm 0.24$ \\
MCHC & $\mathrm{g} / \mathrm{dL}$ & $31.07 \pm 0.40$ & $31.45 \pm 0.37$ & $31.05 \pm 0.42$ & $31.33 \pm 0.25$ \\
HGB & $\mathrm{g} / \mathrm{dL}$ & $13.13 \pm 0.10$ & $13.73 \pm 0.21$ & $13.22 \pm 0.33$ & $13.70 \pm 0.32$ \\
PLT & $\mathrm{X} 10^{3} \mu \mathrm{L}$ & $1373.83 \pm 160.27$ & $1383.83 \pm 30.84$ & $1424.17 \pm 71.48$ & $1336.33 \pm 93.97$ \\
WBC & $\mathrm{X} 10^{3} \mu \mathrm{L}$ & $12.97 \pm 1.37$ & $12.99 \pm 1.51$ & $11.91 \pm 1.32$ & $12.14 \pm 0.86$ \\
Differential & Neutrophils & $22.25 \pm 3.19$ & $28.92 \pm 2.64$ & $36.65 \pm 5.83$ & $38.57 \pm 2.79$ \\
WBCs & Lymphocytes & $73.30 \pm 3.35$ & $71.35 \pm 2.75$ & $65.08 \pm 5.24$ & $61.23 \pm 4.21$ \\
$(\%)$ & Monocytes & $1.47 \pm 0.21$ & $1.90 \pm 0.10$ & $2.03 \pm 0.27$ & $2.70 \pm 0.42$ \\
& Eosinophils & $0.70 \pm 0.09$ & $0.87 \pm 0.13$ & $0.82 \pm 0.06$ & $0.77 \pm 0.11$ \\
& Basophils & $0.40 \pm 0.04$ & $0.50 \pm 0.07$ & $0.37 \pm 0.06$ & $0.45 \pm 0.08$ \\
\hline
\end{tabular}

$>$ Values are given as mean + SEM for groups of 6 animals each.

$>*_{p}<0.05$ as compared to vehicle control was considered as significant.

BIOCHEMICAL ANALYSIS: The result data of biochemical study are depicted in table 5 and 6, in male and female animals; respectively. The serum AST, ALT, total cholesterol, triglycerides, creatinine, total bilirubin, and total proteins including albumin and globulin level does not show any significant variation in animals treated with different doses of dexamethasone palmitate injection as compared to control treated group. The reason for increase in ALP level may be due to altered hepatic metabolism which contributing prolonged induction of ALP production by the liver [25]. At the same time, marked increase in glucose levels was observed, which is one of the adverse effect attributed to dexamethasone. The previous study has reported that treatment with dexamethasone increases glucose level by promoting hepatic gluconeogenesis and inhibit its peripheral utilization [26]. The waste product of protein metabolism, urea is eliminated through renal route. The increased serum urea indicates functional damage in kidney but histopathological examination revealed no treatment related toxic changes in kidneys.

Table 5: Effect of Dexamethasone Palmitate on biochemical parameters in male wistar rats

\begin{tabular}{lcccc}
\hline \multicolumn{1}{c}{ Parameters } & Group I & Group II & Group III & Group IV \\
\hline AST ( IU/L) & $245.02 \pm 16.80$ & $222.90 \pm 21.50$ & $268.61 \pm 36.86$ & $234.66 \pm 9.46$ \\
ALT ( IU/L) & $36.74 \pm 1.86$ & $38.35 \pm 3.86$ & $39.04 \pm 3.78$ & $32.94 \pm 2.16$ \\
ALP ( IU/L) & $205.17 \pm 10.42$ & $219 \pm 14.27$ & $236.33 \pm 26.63$ & $249.00 \pm 6.25$ \\
Total Protein (g/dL) & $7.17 \pm 0.03$ & $7.58 \pm 0.11$ & $7.42 \pm 0.22$ & $7.44 \pm 0.05$ \\
Albumin (g/dL) & $3.45 \pm 0.03$ & $3.76 \pm 0.07$ & $3.67 \pm 0.10$ & $3.84 \pm 0.06$ \\
Globulin (g/dL) & $3.72 \pm+0.01$ & $3.82 \pm 0.07$ & $3.75 \pm 0.21$ & $3.40 \pm 0.06$ \\
Glucose (mg/dL) & $49.00 \pm 6.49$ & $73.00 \pm 11.96$ & $79.00 \pm 8.38$ & $88.33 \pm 5.56$ \\
Cholesterol (mg/dL) & $48.00 \pm 2.72$ & $48.81 \pm 1.80$ & $48.21 \pm 4.70$ & $48.83 \pm 1.99$ \\
Triglycerides(mg/dL) & $69.67 \pm 5.77$ & $74.17 \pm 9.92$ & $73.67 \pm 4.57$ & $67.17 \pm 13.52$ \\
Urea (mg/dL) & $36.20 \pm 0.99$ & $43.32 \pm 1.35$ & $48.41 \pm 4.25$ & $54.81 \pm 1.13$ \\
Creatinine (mg/dL) & $0.45 \pm 0.01$ & $0.47 \pm 0.02$ & $0.48 \pm 0.02$ & $0.43 \pm 0.02$ \\
Total Bilirubin (mg/dL) & $0.32 \pm 0.00$ & $0.31 \pm 0.01$ & $0.32 \pm 0.01$ & $0.30 \pm 0.01$ \\
\hline
\end{tabular}

$>$ Values are given as mean + SEM for groups of 6 animals each.

$>*_{\mathrm{p}}<0.05$ as compared to vehicle control was considered as significant. 
Table 6: Effect of dexamethasone palmitate on biochemical parameters in female wistar rats

\begin{tabular}{lcccc}
\hline \multicolumn{1}{c}{ Parameters } & Group I & Group II & Group III & Group IV \\
\hline AST ( IU/L) & $212.94 \pm 32.35$ & $223.19 \pm 9.49$ & $202.80 \pm 8.80$ & $217.16 \pm 19.55$ \\
ALT ( IU/L) & $38.49 \pm 4.49$ & $33.23 \pm 2.26$ & $36.88 \pm 5.15$ & $32.52 \pm 1.43$ \\
ALP ( IU/L) & $140.33 \pm 18.61$ & $144.17 \pm 11.21$ & $153.33 \pm 17.68$ & $162.50 \pm 12.21$ \\
Total Protein (g/dL) & $7.19 \pm 0.20$ & $7.82 \pm 0.17$ & $7.93 \pm 0.23$ & $7.71 \pm 0.10$ \\
Albumin (g/dL) & $3.68 \pm 0.14$ & $3.12 \pm 0.12$ & $3.92 \pm 0.19$ & $3.92 \pm 0.08$ \\
Globulin (g/dL) & $3.51 \pm 0.12$ & $3.70 \pm 0.09$ & $3.91 \pm 0.14$ & $3.79 \pm 0.08$ \\
Glucose (mg/dL) & $56.67 \pm 8.58$ & $63.00 \pm 4.41$ & $72.00 \pm 5.78$ & $79.50 \pm 5.00$ \\
Cholesterol (mg/dL) & $44.50 \pm 4.33$ & $41.33 \pm 3.42$ & $42.67 \pm 3.79$ & $44.33 \pm 4.92$ \\
Triglycerides (mg/dL) & $51.67 \pm 2.88$ & $58.17 \pm 7.50$ & $54.00 \pm 10.37$ & $57.17 \pm 8.35$ \\
Urea (mg/dL) & $41.83 \pm 2.19$ & $46.98 \pm 1.84$ & $48.14 \pm 3.24$ & $51.21 \pm 1.75$ \\
Creatinine (mg/dL) & $0.53 \pm 0.01$ & $0.58 \pm 0.03$ & $0.53 \pm 0.03$ & $0.49 \pm 0.02$ \\
Total Bilirubin (mg/dL) & $0.18 \pm 0.01$ & $0.10 \pm 0.01$ & $0.16 \pm 0.01$ & $0.12 \pm 0.01$ \\
\hline
\end{tabular}

$>$ Values are given as mean + SEM for groups of 6 animals each.

$>*_{\mathrm{p}}<0.05$ as compared to vehicle control was considered as significant.

Effect on organ weight and necropsy: No significant changes; in weight of different isolated organs was recorded. The result data of study in male and female animals are depicted in table 7. At the same time necropsy examination also not revealed any external as well as internal pathological findings in sacrificed animals. Some what impact on decreased thymus size was observed in male and female animals with higher dose. The previous study has demonstrated that glucocorticoids in high dose cause thymocyte apoptosis [27-28].

Table7: Effect of dexamethasone palmitate on individual organ weight in male wistar rats

\begin{tabular}{ccccccccc}
\hline Organ & \multicolumn{9}{c}{ Weight in gram (G) } \\
\cline { 2 - 9 } & \multicolumn{2}{c}{ Group I } & \multicolumn{2}{c}{ Group II } & \multicolumn{2}{c}{ Group III } & \multicolumn{2}{c}{ Group IV } \\
\hline \multirow{3}{*}{ Male } & Female & Male & Female & Male & Female & Male & Female \\
Liver & $9.51 \pm 0.27$ & $6.64 \pm 0.38$ & $9.26 \pm 0.23$ & $6.44 \pm 0.44$ & $9.72 \pm 0.29$ & $6.26 \pm 0.30$ & $8.89 \pm 0.42$ & $6.69 \pm 0.42$ \\
Heart & $1.26 \pm 0.06$ & $0.89 \pm 0.03$ & $1.14 \pm 0.02$ & $0.79 \pm 0.03$ & $1.29 \pm 0.02$ & $0.85 \pm 0.04$ & $1.10 \pm 0.02$ & $0.90 \pm 0.06$ \\
Spleen & $0.48 \pm 0.4$ & $0.51 \pm 0.02$ & $0.49 \pm 0.2$ & $0.44 \pm 0.03$ & $0.41 \pm 0.2$ & $0.48 \pm 0.03$ & $0.42 \pm 0.3$ & $0.41 \pm 0.02$ \\
Lungs & $1.73 \pm 0.11$ & $1.18 \pm 0.05$ & $1.47 \pm 0.05$ & $1.09 \pm 0.07$ & $1.75 \pm 0.36$ & $1.19 \pm 0.07$ & $1.35 \pm 0.02$ & $1.12 \pm 0.04$ \\
Adrenals & $0.06 \pm 0.00$ & $0.07 \pm 0.01$ & $0.06 \pm 0.00$ & $0.06 \pm 0.00$ & $0.05 \pm 0.00$ & $0.06 \pm 0.00$ & $0.04 \pm 0.00$ & $0.05 \pm 0.00$ \\
Brain & $1.97 \pm 0.04$ & $1.84 \pm 0.05$ & $1.99 \pm 0.04$ & $1.90 \pm 0.05$ & $1.88 \pm 0.04$ & $1.86 \pm 0.03$ & $1.76 \pm 0.05$ & $1.85 \pm 0.05$ \\
Kidneys & $2.12 \pm 0.05$ & $1.44 \pm 0.03$ & $2.01 \pm 0.05$ & $1.44 \pm 0.55$ & $1.93 \pm 0.1$ & $1.38 \pm 0.04$ & $1.99 \pm 0.04$ & $1.27 \pm 0.05$ \\
\hline
\end{tabular}

$>$ Values are given as mean + SEM for groups of 6 animals each.

$>*_{\mathrm{p}}<0.05$ as compared to vehicle control was considered as significant.

\section{HISTOPATHOLOGICAL FINDINGS}

Histopathology of all organs showed no abnormalities in both sexes in all groups except few below mentioned organs.

Thymus: Histopathology of thymus showed individual cell necrosis, cortex, multifocal in high dose treated group (IV) of animals on using H \& E stain with $200 \times$ magnifications. 


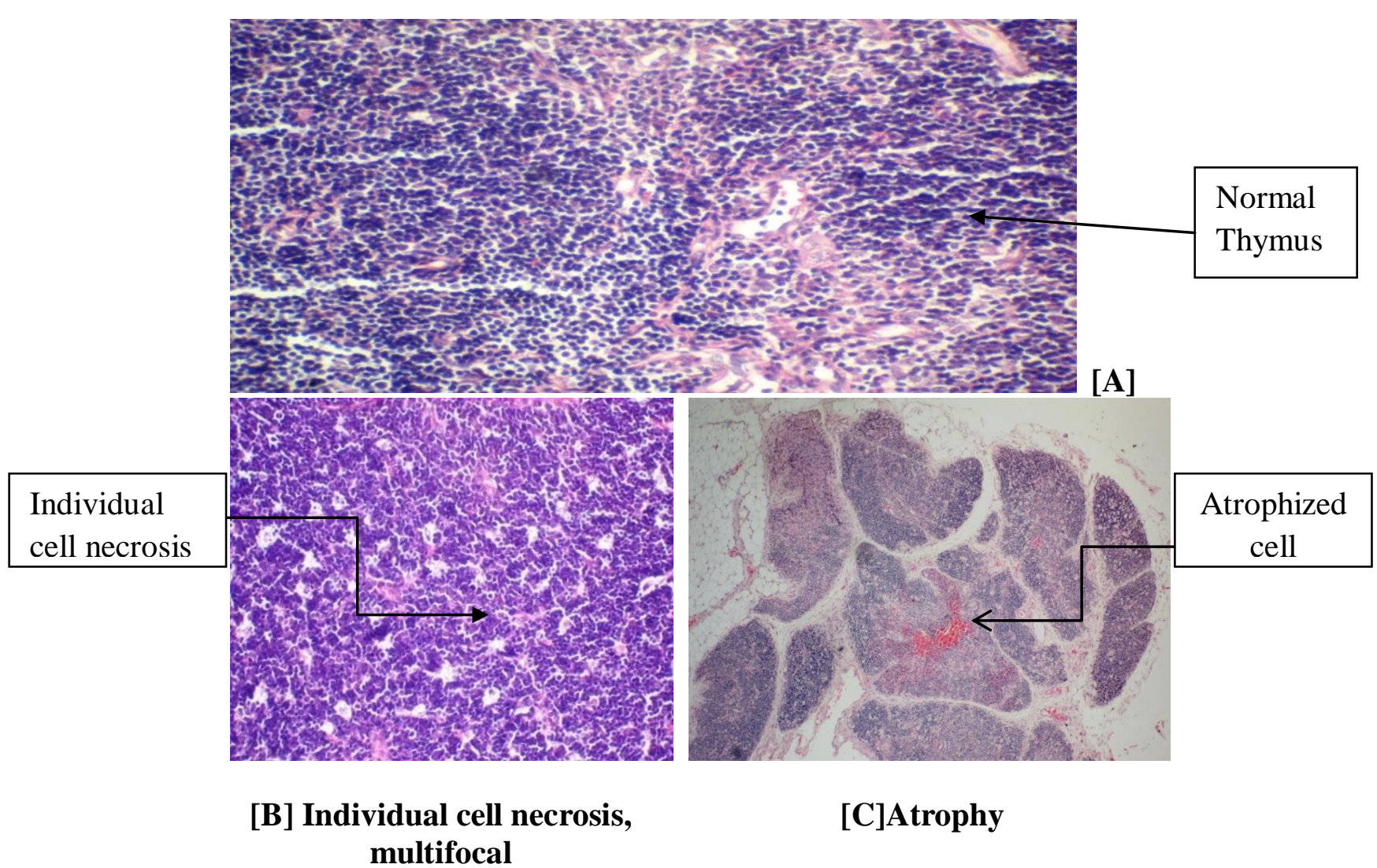

Figure1. Histopathology of thymus [Normal; A] revealed, Individual cell necrosis [B] and atrophy [C] in high dose treated animals

Mesenteric Lymph Node: Histopathology of mesenteric lymph node revealed decreased lymphoid cell population in animals of high dose treated group (Group IV) [B] as compared to vehicle group (Group I) [A].

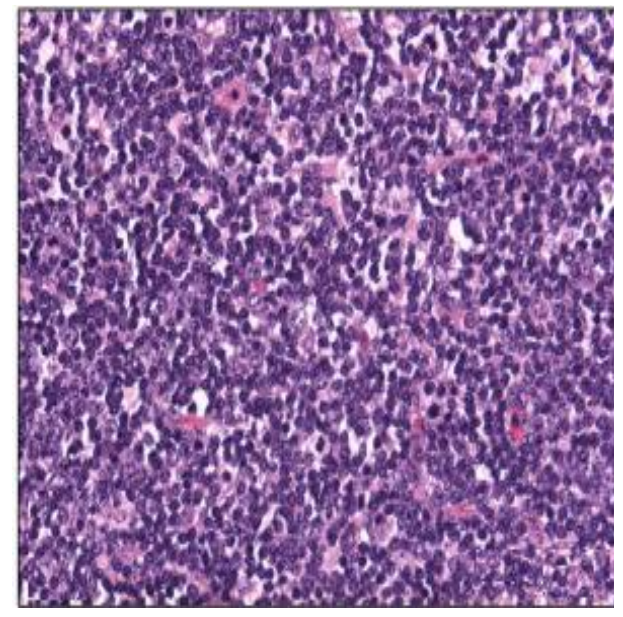

Normal

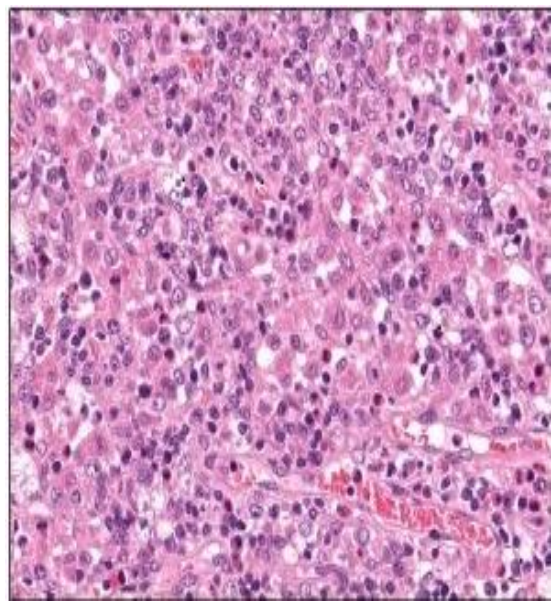

Decreased lymphoid cell count

Figure 2: Histopathological findings of mesenteric lymph node in Wistar rats

Kidneys: Histopathology of kidneys revealed increased MNC infiltration in high dose treated group (Group IV) [B] as compared to vehicle group (Group I) [A]. 


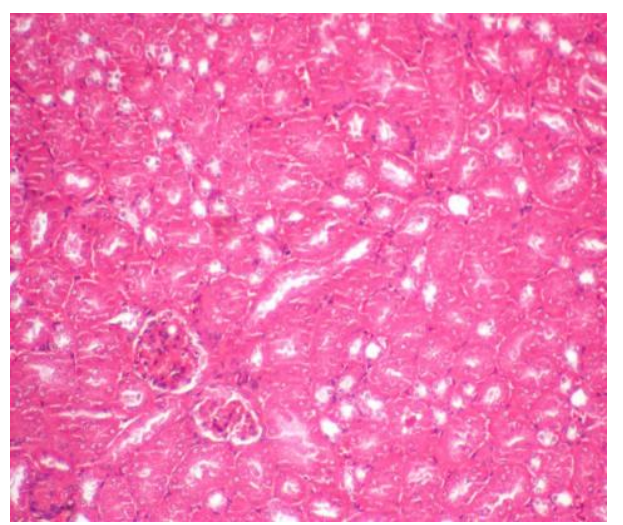

[A]Normal Kidney

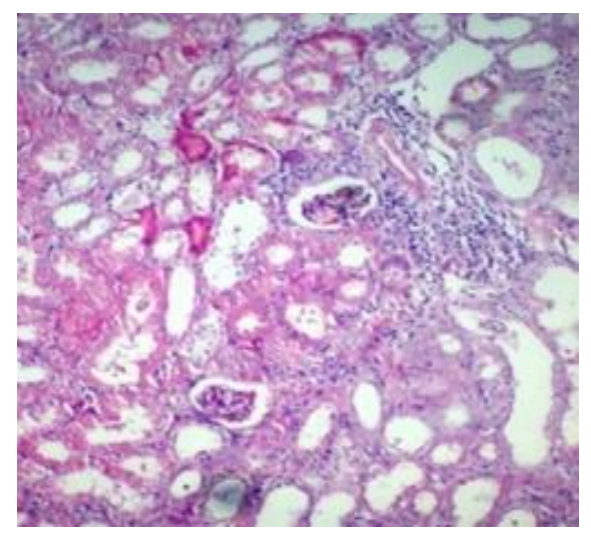

[B]Kidney with MNC infiltration

Figure 3: Histopathological findings of kidney

Uterus: Histopathology of uterus revealed dilated lumen in female group (IV) treated with high dose [B] as compared to vehicle treated group (I) [A].

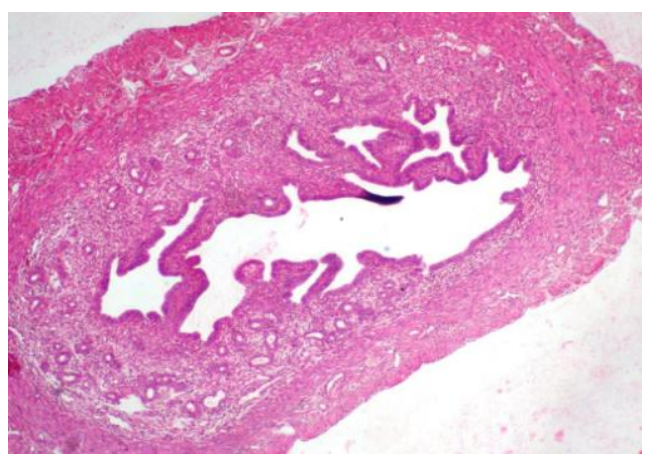

[A]Normal

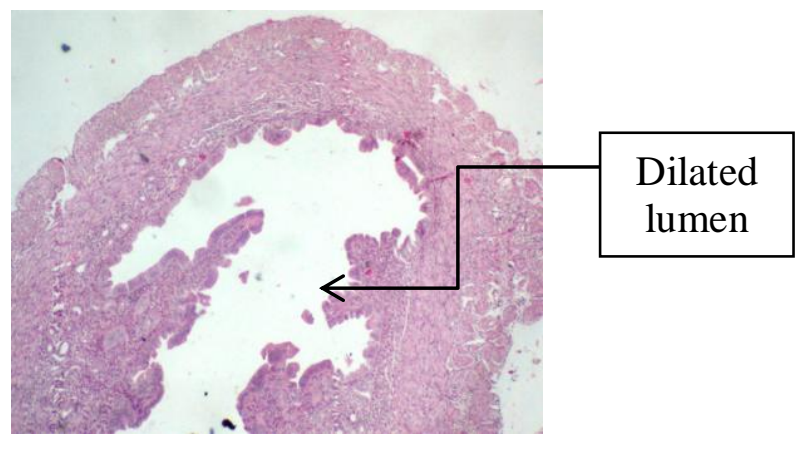

[B]Uterus with dilated lumen

Figure 4: Histopathological findings of Uterus

\section{CONCLUSION}

The study generated data revealed that the intravenous administration of dexamethasone palmitate causes inhibitory effect on normal growth of the animals and inhibit the rate of increase in body weight, although the feed consumption capacity of animals remains unchanged. An increase in total RBCs count, neutrophills and monocytes were also found, while the lymphocyte counts were found to decline in dose dependant manner in drug treated animals. All above mentioned cellular count changes were in reference laboratory range and were not significant, hence were not counted as adverse effect. No significant alteration in other blood cells was recorded. The biochemical analysis also demonstrate the safety margin of the dexamethasone palmitate injection in experimental animals, as no significant changes were observed in biochemical parameters of drug treated animals as compared to compared animals, although somewhat increased ALP and glucose levels was observed. The histological examinations of various organs of experimental animals revealed the somewhat toxicity due to drug, which was seen only at higher dose. The overall study established the safety profile of dexamethasone palmitate against induced sub acute toxicity on intravenous administration. However, a conclusive remark can only be made on the safety profile of drug after further investigations of the observations made in the study at the cellular level in chronic study. 
ACKNOWLEDGEMENT: The author(s) are thankful to Cadila Pharmaceuticals Pvt. Ltd (Dholka), Ahmadabad, Gujrat (India) for providing necessary support to carry out this research work.

\section{REFERENCES}

1. Tortora, GJ.; Derrickson, B., Principles of Anatomy and Physiology, 11: 642-643, 1998.

2. Goyal, RK., Elements of Pharmacology, B S shah Publication, 7: 183, 2007.

3. Walker, R.; Whittlesea, C., Clinical Pharmacy and Therapeutics, 4: 365, 2007.

4. Miyoshi H., Ohki M., Nakagawa T., Honma Y. Glucocorticoids induce Apoptosis in acute myeloid leukaemia cell lines with at $(8 ; 21)$ chromosome translocation. Leuk. Res, 21: 45-50,1997.

5. Trevor, AJ.; Katzung, BG., Pharmacology. Tata McGraw Hill, Newyork Publication, 7: 903, 2002.

6. Collaborative Dexamethasone Trial: Dexamethasone therapy in neonatal chronic lung disease: an international placebo-controlled trial, Pediatrics, 88: 421-427, 1991.

7. Cummings, JJ.; D'Eugenio, DB.; Gross SJ. A controlled trial of Dexamethasone in preterm infants at high risk for bronchopulmonary dysplasia, New Engl. J. Med, 320:1505-1510,1989.

8. Kraaij, MD.; Kooij, SWV.; Reinders, ME.; Koekkoek, K.; Rabelink, TJ.; Kooten, CV. Dexamethasone increases ROS production and $\mathrm{T}$ cell suppressive capacity by antiinflammatory macrophages, Molecular immunology, 49: 549-557, 2011.

9. Motyka, B.; Bhopal, HS. ; Reynolds, JD. Apoptosis of ileal Peyer's patch B cells is increased by glucocorticoids or anti-immunoglobulin antibodies, Eur. J. Immunol, 25: 1865-1871, 1995.

10. Walum, E., Environ Health Perspect,; 106 (2): 497-503, 1998.

11. Goyal, RK., Practicals in Pharmacology, B S Shah Publication, 7: 148-149, 2007.

12. Katzung, BG., Basic and Clinical Pharmacology, MC Graw-Hill Medical Publishing Division, 10: 1065, 2006.

13. Kumari, M.; Mukherjee, A.; Chandrasekaran, N. Genotoxicity of silver nanoparticles in Allium cepa, Science of The total Environment, 407 (19): 5243-5246, 2009.

14. Allen, DB.; Mullen, M.; Mullen, B. A meta-analysis of the effect of oral and inhaled corticosteroids on growth, J Allergy Clin Immunol, 94: 967-976, 1994.

15. Michel, C.; Cabanac, M. Effects of dexamethasone on the body weight set point of rats, Physiology \& behavior, 68: 145-50, 1999.

16. Avioli, LV. Glucocorticoid effects of statural growth, Br J Rheum, 21: 27-30, 1993.

17. Jux, C.; Leiber, K.; Hugel, U.; Blum, W.; Ohlsson, C.; Klaus, G.; Mehls, O. Dexamethasone impairs growth hormone $(\mathrm{GH})$-stimulated growth by suppression of local insulin-like growth factor (IGF)-1 production and expression of GH- and IGF-1 receptor in cultured rat chondrocytes, Endocrinology,139: 3296-3305, 1998.

18. Klaus, G.; Jux, C.; Fernandez, P.; Rodriguez, J.; Himmele, R.; Mehls, O. Suppression of growth plate chondrocyte proliferation by corticosteroids, Pediatr Nephrol,14: 612-615, 2000.

19. Brownlee, KG.; Ng , PC.; Henderson, MJ.; Smith, M.; Green, JH.; Dear, PRF. Catabolic effect of dexamethasone in the preterm baby, Arch.Dis.Child, 61: 1-4, 1992.

20. Malgor, LA.; Torales, PR.; Klainer, E.; Barrios, L.; Blanc, CC. Effects of Dexamethasone on bone marrow erythropoiesis, Horm. Res. (Basel), 5: 269-277,1974.

21. Meints, RH.; Carver, FJ. Triiodothyronine and hydrocortisone effects on Rana pipiens erythropoiesis, Gen. Comp. Endocrinoogy,21: 9-15, 1973.

22. Kufe, DW.; Pollock, RE.; Weichselbaum, RR.; Bast, RC.; Gansler, TS.; Holland, JF. Physiologic and Pharmacologic Effects of Corticosteroids, 43: 314-329, 2003.

23. Leekumjorn, S.; Wu, Y.; Sum, AK.; Chan, C. Experimental and computational studies investigating trehalose protection of HepG2 cells from palmitate-induced toxicity, Biophysical Journal,; 94 (7): 2869-2883, 2008. 
24. Ohkaru, Y.; Arai, N.; Ohno, H.; Sato, S.; Sakakibara, Y.; Suzuki, H.; Aritoshi, S.; Akimoto, S.; Ban, T.; Tanihata, J.; Tachiyasiki, K.; Imaizumi, K. Acute and subacute effects of dexamethasone on the number of white blood cells in rats, Journal of health science, 56: 215220, 2010.

25. Ginel, PJ.; Lucena, R.; Fernandez, M. Duration of increased serum alkaline phosphatase activity in dogs receiving different glucocorticoid doses, Research in veterinary science, 72:201-204, 2002.

26. Katheeri, NA.; Wasfi, I.; Lambert, M.; Saeed, A. Pharmacokinetics and pharmacodynamics of dexamethasone after intravenous administration in camels: effect of dose, Veterinary research communications, 28: 525-542, 2004.

27. Cifone, MG.; Migliorati, G.; Parroni, R.; Marchetti, C.; Millimaggi, D.; Santoni, A.; Riccardi, A. Dexamethasone-induced thymocyte apoptosis: apoptotic signal involves the sequential activation of phosphoinositide-specific phospholipase $\mathrm{C}$, acidic sphingomyelinase, and caspases, Blood, 93:2282-2296, 1999.

28. Ichiyoshi, H.; Kiyozuka, Y.; Kishimoto, Y.; Fukuhara, S.; Tsubura, A. Massive telomerase loss and telomerase RNA expression in dexamethasone-induced apoptosis in mouse thymocytes, Experimental and molecular pathlogy, 7: 178-186, 2003. 